\title{
MODELO DE UN GRADO DE LIBERTAD PARA EVALUAR LA CURVA CARGA LATERAL-DISTORSIÓN EN MUROS DE MAMPOSTERÍA CONFINADA
}

\author{
Sulpicio Sánchez ${ }^{(1)}$, Roberto Arroyo $^{(1)}$, Sandra Jerez $^{(2)}$
}

\section{RESUMEN}

Este artículo presenta un modelo simple para evaluar la curva de carga lateral-distorsión en ensayes de muros de mampostería confinada. El modelo propone sustituir la mampostería por un elemento resorte, cuyas propiedades mecánicas son obtenidas mediante pruebas en paneles de mampostería sujetos a tensión diagonal. De esta manera, un muro de mampostería (integrado por castillos y mampostería) es modelado como un sistema equivalente de un grado de libertad con desplazamiento lateral, cuya respuesta es obtenida mediante un análisis estático no lineal, considerando exclusivamente deformaciones de cortante. La comparación con resultados experimentales obtenidos en México para muros y sistemas estructurales ensayados bajo carga cíclica lateral muestran la capacidad del modelo, que tiene aproximación aceptable hasta una distorsión igual a $0.45 \%$ para muros elaborados con tabique rojo recocido y $0.35 \%$ para muros elaborados con piezas de concreto. Ambos valores son mayores que el permisible definido en el Reglamento del Distrito Federal. Adicionalmente, los resultados tienen una aproximación similar a la obtenida en modelos complejos de elemento finito, columna ancha y puntales de compresión.

Palabras Clave: mampostería confinada; deformación de cortante; sistema equivalente; carga lateral

\begin{abstract}
This paper shows a simple model to evaluate the backbone curve of confined masonry walls (CMW) tested under lateral load. In this way, the shear stress vs. shear strain relationship obtained from the diagonal tension tests is used to model the masonry behavior by means of a spring element. Then, a single degree of freedom equivalent system, which takes account only the shear horizontal deformations, represents the concrete tie-column and masonry elements. A non-linear static analysis is developed in order to evaluate the backbone curve. Experimental results of CMW and structural systems tested in Mexico are used to compare the numeric results. Thus, the model has an adequate agreement until a distortion equal to $0.45 \%$ or $0.35 \%$ for CMW built from solid clay bricks or concrete blocks, respectively. Both values are greater than the permissible distortion proposed by the Mexico City Code. Besides, the results agreement is similar to that obtained from numerical models developed by means of the finite element method, wide column model, or equivalent strut model.
\end{abstract}

Artículo recibido el 12 de enero de 2010 y aceptado para su publicación el 5 de noviembre de 2010. Se aceptarán comentarios y/o discusiones hasta cinco meses después de su publicación

(1) Universidad Autónoma de Guerrero. Av. Lázaro Cárdenas s/n. Ciudad Universitaria. Col La Haciendita, 39000, Chilpancingo, Gro, México. Email: sstizapa@hotmail.com, arroyomatus@hotmail.com

(2) Escuela Colombiana de Ingeniería. AK.45 No.205-59, Autopista Norte, A.A. 14520, Bogotá, DC, Colombia. Email: sjerez2004@yahoo.com 
Key Words: confined masonry walls; diagonal tension; backbone curve; solid clay brick; distortion

\section{INTRODUCCIÓN}

Los muros de mampostería confinada son utilizados en la construcción del 70\% de viviendas y/o edificios habitacionales en México (INEGI, 2005; Rodríguez, 2009). Sin embargo, las piezas utilizadas pueden presentar una variación de hasta $40 \%$ en sus propiedades mecánicas (Sánchez, 2009; Meli y Hernández, 1971) dificultando la evaluación de la seguridad estructural de las edificaciones construidas con este material, especialmente en la costa del Pacífico mexicano donde las fuerzas sísmicas laterales pueden representar hasta $43 \%$ del peso de las construcciones (GEG, 1989).

Por lo anterior, es importante contar con herramientas simples que permitan evaluar la envolvente de respuesta de los muros de mampostería sujetos a carga lateral. Para garantizar su utilidad y evitar ensayes de muros a escala real, en zonas donde no existen instalaciones adecuadas, dichas herramientas deben basarse en propiedades mecánicas evaluadas en pruebas de especímenes a escala reducida (pilas y/o muretes) elaboradas con las mismas piezas utilizadas en la construcción de los muros.

Una propuesta de este tipo, basada en el esfuerzo cortante obtenido en la prueba de tensión diagonal, ha sido presentada por Riahi, Elwood y Alcocer (2009). Los autores realizaron un análisis estadístico de las curvas esfuerzo cortante-distorsión para un extenso tipo de ensayes de muros elaborados con piezas de arcilla y concreto. Así, ellos propusieron una curva de comportamiento tri-lineal definida por tres pares de valores esfuerzo cortante y distorsión, los cuales están asociados al agrietamiento, máximo esfuerzo cortante y distorsión última. La aplicación del modelo requiere la relación altura/longitud del muro, esfuerzo vertical actuante, resistencia a compresión de la mampostería y concreto así como las propiedades del acero transversal.

A diferencia del modelo anterior calibrado de forma estadística, el modelo aquí desarrollado evalúa la curva envolvente carga lateral - distorsión a partir de la mecánica de materiales y está basado en la siguiente información:

1. Comportamiento de muros de mampostería referenciados por Aguilar (1997), Flores (1995), Hernández y Urzua (2002), Sánchez (2009) y Treviño et al. (2004),

2. Curva esfuerzo cortante-deformación de cortante obtenida en pruebas de tensión diagonal según Sánchez (2009),

3. Evaluación de la carga resistente lateral según Sánchez y Mebarki (2009).

De esta forma, el muro confinado sujeto a carga lateral y esfuerzo vertical mostrado en la Figura 1a puede modelarse según la Figura $1 \mathrm{~b}$, donde $\boldsymbol{k}_{\boldsymbol{M}}$ es la rigidez de la mampostería sobre la diagonal y $\boldsymbol{k}_{\boldsymbol{C}}$ es la rigidez lateral de los castillos. Considerando que el muro sufre exclusivamente deformaciones de cortante generadas por la carga lateral, el sistema puede modelarse como un sistema con elementos en paralelo y un solo grado de libertad representado por el desplazamiento lateral, Figura 1c. El modelo supone la falla simultánea de ambos castillos cuando se alcanza la fuerza cortante asociada al momento de fluencia.

\section{COMPORTAMIENTO DE LA MAMPOSTERÍA}

Las propiedades mecánicas del elemento resorte, utilizado para modelar la mampostería, son evaluadas a partir de las curvas esfuerzo cortante-deformación tangencial registradas en pruebas de tensión diagonal. La Figura 2, obtenida en muretes elaborados con tabique rojo recocido (Sánchez, 2009), muestra que la respuesta puede representarse por una curva con comportamiento lineal hasta el esfuerzo 
máximo $\boldsymbol{v}_{\boldsymbol{m}}$ asociado a la deformación tangencial $\gamma_{\boldsymbol{m}}$, mientras que el segundo punto es definido por el esfuerzo máximo $\boldsymbol{v}_{\boldsymbol{m}}$ y la deformación tangencial última, $\boldsymbol{\gamma}_{u}$, ver Tabla 1, según Sánchez (2009).

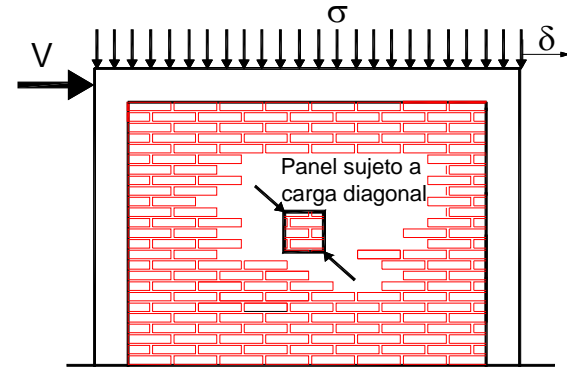

a)

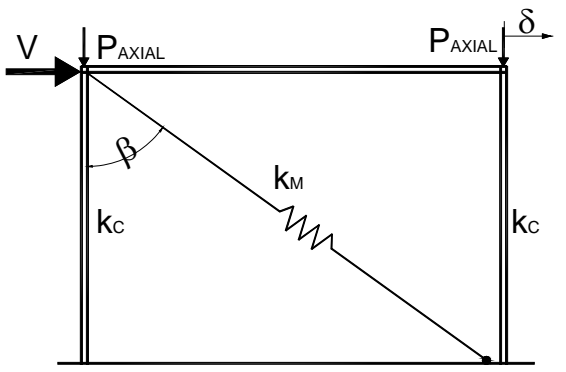

b)

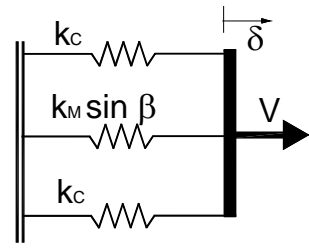

c)

Figura1: a) Muro de mampostería sujeto a carga lateral y vertical, b) Macro-modelo, c) Modelo simplificado de un grado de libertad con deformación de cortante

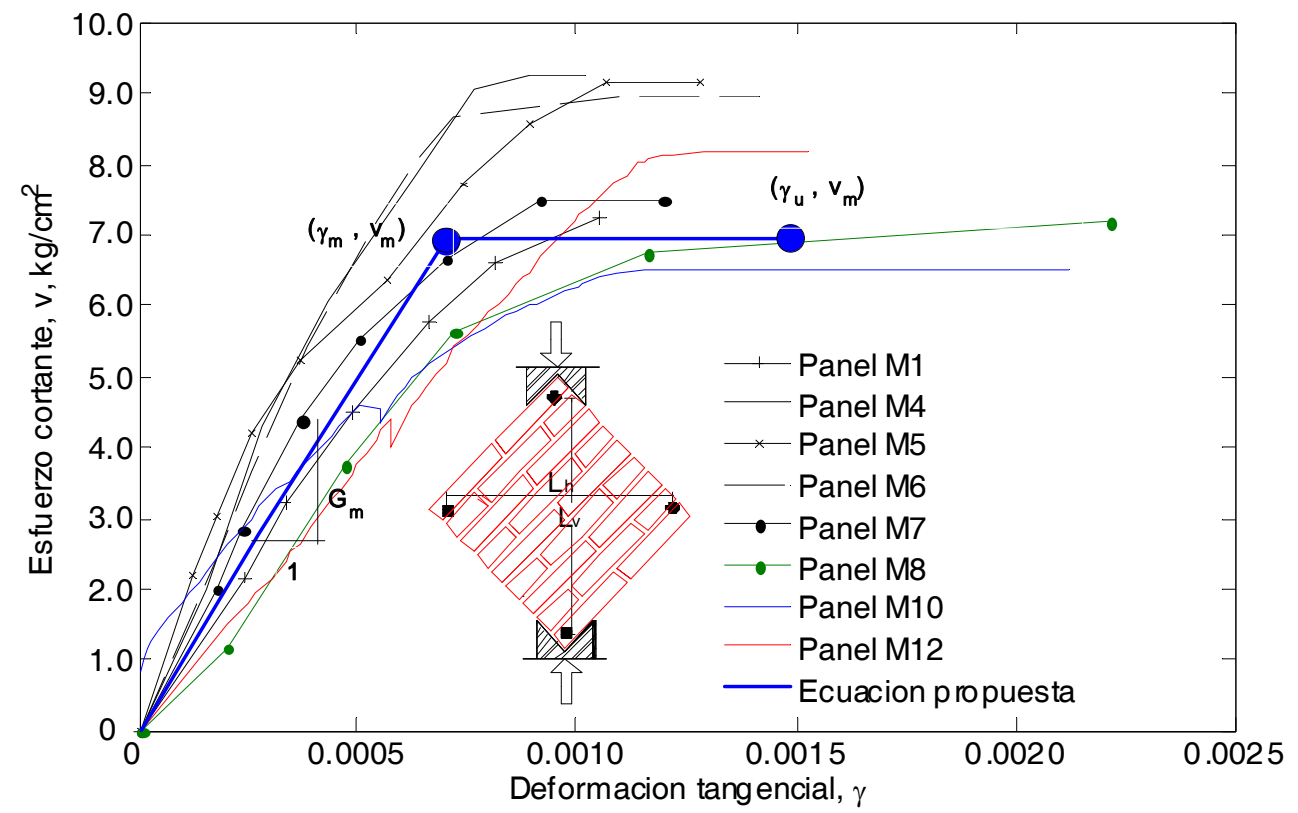

Figura 2: Curvas experimentales en pruebas de tensión diagonal y curva propuesta (Sánchez, 2009)

Tabla 1 Propiedades mecánicas de la mampostería (Sánchez, 2009)

\begin{tabular}{ccccc}
\hline Parámetro & $\boldsymbol{v}_{\boldsymbol{m}},\left[\mathrm{kg} / \mathrm{cm}^{2}\right]$ & $\boldsymbol{G}_{\boldsymbol{m}},\left[\mathrm{kg} / \mathrm{cm}^{2}\right]$ & $\gamma_{\boldsymbol{m}}$ & $\gamma_{u l t}$ \\
\hline Valor medio & 7.1 & 10130 & 0.0007 & 0.0015 \\
\hline
\end{tabular}

En otro orden, según los resultados experimentales referenciados por Sánchez (2009) y Gabor et al. (2006), la deformación horizontal en los ensayes de tensión diagonal es despreciable respecto a la deformación vertical. Por lo tanto, el acortamiento vertical $\boldsymbol{\delta}_{\boldsymbol{v}}$ de la longitud de control $\boldsymbol{L}_{\boldsymbol{v}}$, correspondiente a un valor de deformación tangencial $\gamma_{v i}$, está definido por la Ecuación uno (ASTM 2007). En el rango 
lineal, la deformación tangencial $\gamma_{v i}$ es obtenida mediante la Ecuación dos, donde $\boldsymbol{G}_{\boldsymbol{m}}$ es el módulo de corte de la mampostería. Por otro lado, la carga actuante es definida por la Ecuación tres, donde $\boldsymbol{A}_{\boldsymbol{d}}$ es el área diagonal del panel y $\boldsymbol{v}_{\boldsymbol{i}}$ es el esfuerzo cortante asociado a la deformación tangencial $\boldsymbol{\gamma}_{\boldsymbol{v} i}$. Así, la Figura 3a muestra la curva idealizada carga vertical-desplazamiento vertical para el panel ensayado. Las variables $\boldsymbol{v}$, $\boldsymbol{G}_{\boldsymbol{m}}$ y $\boldsymbol{A}_{\boldsymbol{d}}$ son datos obtenidos en la prueba de tensión diagonal, ver Tabla 1 y Figura 2.

$$
\begin{aligned}
& \delta_{v i}=\gamma_{v i} L_{v} \\
& \gamma_{v i}=v_{i} / G_{m} \\
& V_{i}=v_{i} A_{d}
\end{aligned}
$$

La Figura 3a, obtenida con los resultados de la prueba de tensión diagonal, puede transformarse para obtener la curva de comportamiento del elemento resorte, Figura 3b, cuyos parámetros ya están definidos en la dirección horizontal. Según la Figura 1a y 1b, el desplazamiento de agrietamiento $\boldsymbol{\delta}_{\boldsymbol{a g r}}$ evaluado con la Ecuación uno se proyecta en la dirección horizontal mediante la Ecuación cuatro, donde $\gamma_{\boldsymbol{m}}$ está asociado al esfuerzo cortante $\boldsymbol{v}_{\boldsymbol{m}}$ y es obtenido con la Ecuación dos. Por otro lado y a falta de valores experimentales adicionales fue propuesta la Ecuación cinco, donde $\gamma_{u l t}$ es definida en la Tabla 1. En las Ecuaciones cuatro y cinco $\boldsymbol{L}_{\boldsymbol{d} \boldsymbol{m}}$ y $\boldsymbol{\beta}$ son la longitud de la diagonal del muro y su ángulo respecto a la vertical, ver Figura $1 \mathrm{~b}$.

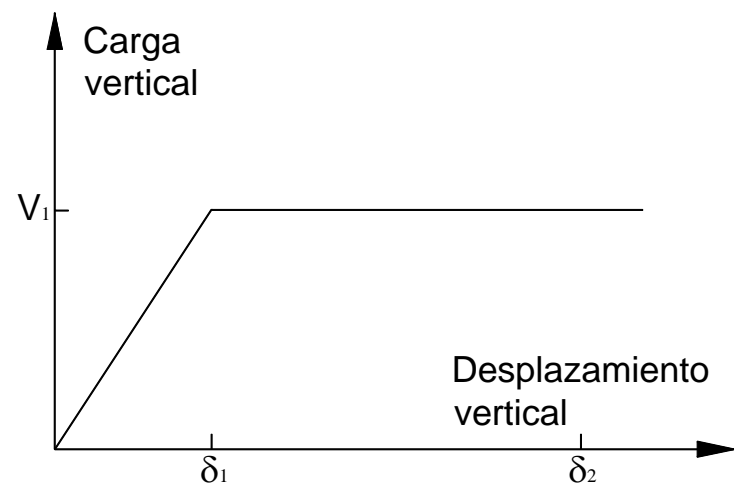

a)

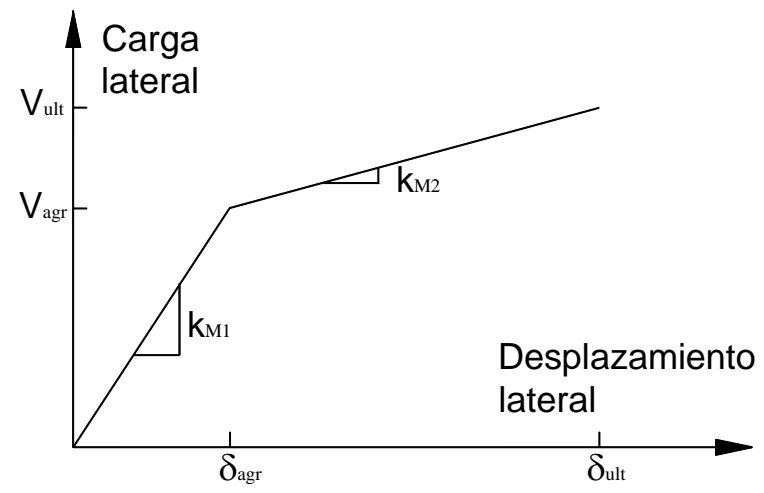

b)

Figura 3: a) Curva experimental carga vertical-desplazamiento vertical en una prueba de tensión diagonal, b) Curva experimental carga lateral-desplazamiento lateral para el elemento diagonal

$$
\begin{aligned}
& \delta_{a g r}=\gamma_{m} L_{d m} \sin (\beta) \\
& \delta_{u l t}=1.25 \gamma_{u l t} L_{d m} \sin (\beta)
\end{aligned}
$$

Por otro lado, la carga de agrietamiento es evaluada mediante la Ecuación seis presentada originalmente por Sánchez y Mebarki (2009) y modificada mediante los factores $\boldsymbol{F}_{\boldsymbol{1}}$ y $\boldsymbol{F}_{\boldsymbol{2}}$ obtenidos mediante calibración experimental en función del tipo de pieza, ver Tabla 2. Los otros términos son: $\sigma$, esfuerzo vertical actuante; $\boldsymbol{A}_{\boldsymbol{d} \boldsymbol{m}}$, área de la diagonal de la mampostería; $\boldsymbol{v}_{\boldsymbol{r}}$ cortante resistente evaluado con 
la Ecuación ocho. El parámetro $\boldsymbol{f}$, evaluado con la Ecuación siete y calibrado experimentalmente, relaciona la rigidez vertical de la mampostería respecto a la rigidez vertical del muro (Sánchez, 2009), donde $\boldsymbol{A}_{\boldsymbol{m}}$ y $\boldsymbol{E}_{\boldsymbol{m}}$ son el área horizontal y módulo elástico de la mampostería mientras que $\boldsymbol{A}_{\boldsymbol{c}}$ y $\boldsymbol{E}_{\boldsymbol{c}}$ son el área y módulo elástico de los castillos. La Ecuación ocho fue obtenida a partir de resultados experimentales de 34 muros elaborados con piezas de concreto y arcilla considerando la ecuación propuesta por Sánchez y Mebarki (2009), ver Apéndice A.

$$
\begin{aligned}
& V_{a g r}=A_{d m} \sin (\beta)\left[F_{1} v_{r}+F_{2} f \sin (\beta) \cos (\beta) \sigma\right] \\
& f=A_{m} E_{m} /\left[A_{m} E_{m}+2 A_{c} E_{c}\right] \\
& v_{r}=\left\{\begin{array}{c}
0.676 v_{m} \text { para piezas de concreto } \\
0.635 v_{m} \text { para piezas de arcilla }
\end{array}\right.
\end{aligned}
$$

Tabla 2 Factores de la Ecuación seis

\begin{tabular}{ccc}
\hline Factor y tipo de pieza & $\boldsymbol{F}_{\boldsymbol{1}}$ & $\boldsymbol{F}_{\boldsymbol{2}}$ \\
\hline Piezas de arcilla & 0.8 & 0.0 \\
Piezas de concreto & 1.0 & 0.1 \\
\hline
\end{tabular}

Finalmente, la carga última de la mampostería $\boldsymbol{V}_{\text {ult }}$ asociada al desplazamiento $\boldsymbol{\delta}_{\boldsymbol{u l t} \text { t }}$ evalúa la aportación de la mampostería y del esfuerzo vertical según la Ecuación nueve (Sánchez y Mebarki, 2009). De esta manera, la rigidez de la curva de comportamiento mostrada en la Figura 3b es calculada con los valores $\boldsymbol{V}_{\text {agr }}, \boldsymbol{\delta}_{\text {agr }}, \boldsymbol{V}_{\text {ult }}$ y $\boldsymbol{\delta}_{\text {ult }}$.

$V_{u l t}=A_{d m} \sin (\beta)\left[v_{r}+f \sin (\beta) \cos (\beta) \sigma\right]$

\section{COMPORTAMIENTO DE LOS CASTILLOS}

Según Tomazevic (2000), la rigidez de los muros de mampostería es la sumatoria de la rigidez del marco de concreto y la mampostería. En este caso, el elemento resorte definido anteriormente proporciona la rigidez de la mampostería; sin embargo, falta por evaluar la rigidez de los castillos. De acuerdo a la Figura 4a obtenida con resultados experimentales, (Sánchez, 2009), la dala superior de concreto no tiene acortamiento axial y se comporta como cuerpo rígido, el cual es representado por el mismo desplazamiento horizontal de ambos extremos mientras que los desplazamientos verticales en los castillos son despreciables. Así, se considera una posición deformada similar a la propuesta por Zúniga y Terán (2008) para el modelo de columna ancha, donde la rigidez lateral de los castillos $\boldsymbol{k}_{\boldsymbol{C}}$ es evaluada con la Ecuación 10, siendo $\boldsymbol{H}$ la altura del muro, Figura 4b.

$k_{C}=12 E I / H^{3}$

En la ecuación 10, el termino $\boldsymbol{E I}$ es variable durante el proceso de carga según la Figura 5. En la primera fase tiene comportamiento lineal hasta el momento de agrietamiento y en la segunda fase se considera la rigidez secante entre el origen y el punto asociado a la fluencia $\left(\boldsymbol{M}_{\boldsymbol{y}}, \boldsymbol{\theta}_{\boldsymbol{y}}\right)$ según Tomazevic 
(2000). El cálculo del diagrama momento-rotación requiere la definición de la ley constitutiva del concreto y del acero, así como de la distribución del acero longitudinal y transversal. En este trabajo fue utilizado el modelo de Kent y Park modificado (Kent y Park, 1971; Ewing y Kowalsky, 2004). Finalmente, la Ecuación 11 evalúa el cortante $\boldsymbol{V}$ asociado al momento de agrietamiento o al de fluencia $\left(\boldsymbol{M}_{\text {agr }}, \boldsymbol{M}_{\boldsymbol{y}}\right.$ ), donde $\boldsymbol{H}$ es la altura de los castillos.

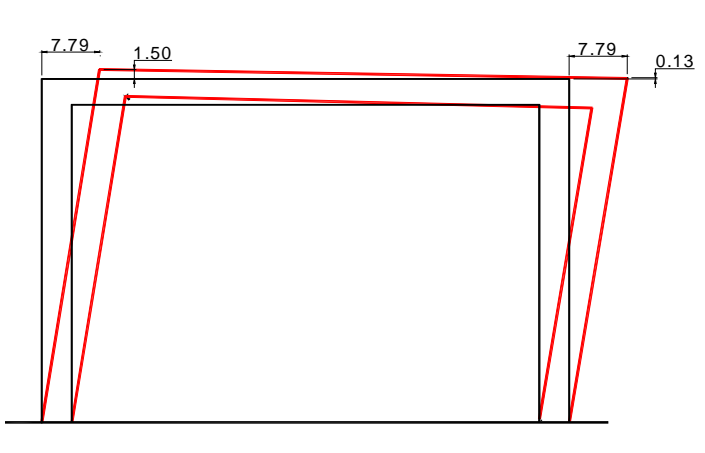

a)

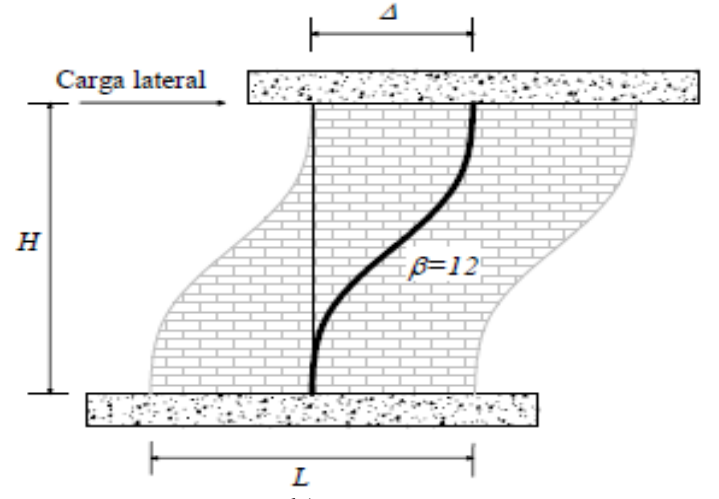

b)

Figura 4: a) Posición deformada [mm] en un muro de mampostería para la carga máxima lateral (Sánchez, 2009), b) Posición deformada propuesta para el modelo de columna ancha (Zúñiga y Terán, 2008)

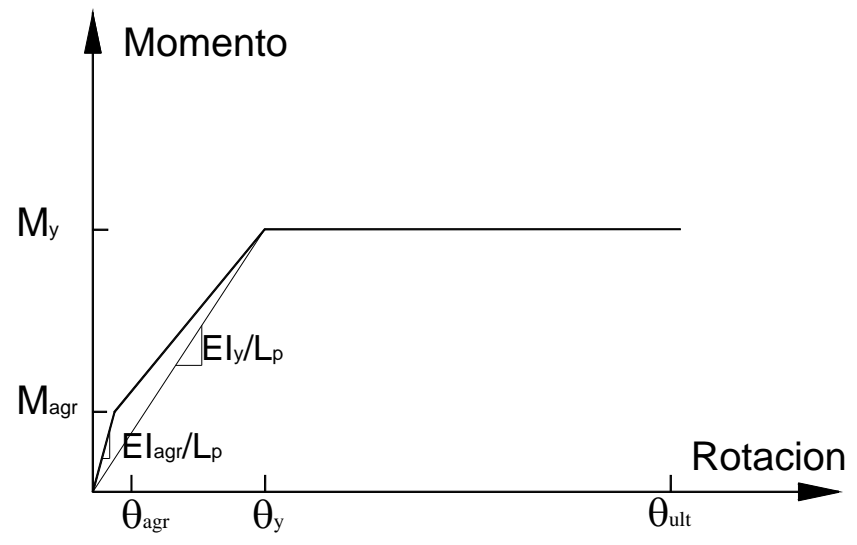

Figura 5: Diagrama momento curvatura de los castillos

\section{PROCEDIMIENTO DE ANÁLISIS}

La principal ventaja del modelo es la simplicidad del análisis como lo muestra la Tabla 5. El proceso puede resumirse en los siguientes pasos (Priestley, Seible y Calvi, 1996):

1. Obtener la matriz de rigidez de la estructura a partir de la rigidez de los elementos en función de la relación rigidez-deformación, Figuras 3b y 5 . Ambos materiales tienen comportamiento lineal en el primer incremento. 
$K_{T}=k_{M}+2 k_{C}$

2. Aplicar una fuerza lateral unitaria,

3. Evaluar el desplazamiento de la estructura,

$\Delta_{U N}=1 / K_{T}$

4. Obtener el cortante en cada elemento asociado a la fuerza unitaria, en la Ecuación $14, \boldsymbol{k}_{\boldsymbol{i}}$ es la rigidez de cada elemento,

$V_{U N}=k_{i} \Delta_{U N}$

5. Calcular el parámetro $\alpha$ para cada elemento, Ecuación 15 , donde $\boldsymbol{V}_{\boldsymbol{S}}$ es el cortante resistente asociado al próximo punto de la curva de capacidad y $\boldsymbol{V}_{T}$ es el cortante total acumulado hasta el paso anterior,

$\alpha=\left(V_{S}-V_{T}\right) / V_{U N}$

6. Evaluar el incremento de cortante $\left(\boldsymbol{\Delta} \boldsymbol{V}_{\boldsymbol{E} L}\right)$ para cada elemento en función del menor valor de $\boldsymbol{\alpha}$ obtenido y actualizar el cortante total $\left(\boldsymbol{V}_{T}\right)$,

$\Delta V_{E L}=\alpha V_{U N}$

$V_{T}=\Sigma \Delta V_{E L}+V_{T}$

7. Evaluar el incremento de desplazamiento $(\boldsymbol{\Delta})$ en función del valor de $\boldsymbol{\alpha}$ elegido y actualizar el desplazamiento total acumulado $\left(\boldsymbol{\Delta}_{\boldsymbol{T}}\right)$,

$\Delta=\alpha \Delta_{U N}$

$\Delta_{T}=\Delta+\Delta_{T}$

8. Regresar al primer paso.

El proceso termina cuando todos los elementos ha alcanzado su capacidad. Finalmente, la curva de capacidad se obtiene al graficar la distorsión contra la fuerza cortante para cada incremento del análisis. La distorsión es la razón desplazamiento lateral (A) y altura $(\boldsymbol{H})$ del muro, Ecuación 20.

$R=\Delta / H$ 


\section{RESULTADOS}

El modelo es aplicado a muros y sistemas estructurales construidos con tabique rojo y a muros construidos con piezas de concreto. La Tabla 3 presenta los datos necesarios para evaluar la rigidez del elemento resorte y el término $\boldsymbol{E I}$ de los castillos según las Figuras 3b y 5. La información experimental fue obtenida de Aguilar (1997), Hernández y Urzua (2002), Sánchez (2009) y Treviño et al. (2004).

Tabla 3 Datos de la mampostería y castillos (ton, m)

\begin{tabular}{c|cccc|cc}
\hline Autor & \multicolumn{3}{|c|}{ Mampostería } & \multicolumn{2}{c}{ Castillos } \\
& $\boldsymbol{V}_{\boldsymbol{a g r}}$ & $\boldsymbol{V}_{\boldsymbol{u l t}}$ & $\boldsymbol{\delta}_{\boldsymbol{a g r}}$ & $\boldsymbol{\delta}_{\boldsymbol{u l t}}$ & $\boldsymbol{E I}_{\boldsymbol{a g r}}$ & $\boldsymbol{E I}_{\boldsymbol{y}}$ \\
\hline Sánchez (2009) & 9.9 & 12.4 & $1.46 \mathrm{E}-3$ & $3.91 \mathrm{E}-3$ & 228.6 & 32.7 \\
Aguilar (1997) & 7.6 & 11.7 & $1.66 \mathrm{E}-3$ & $4.13 \mathrm{E}-3$ & 50.9 & 17.7 \\
Hernández y Urzua (2002) & 8.2 & 10.9 & $0.89 \mathrm{E}-3$ & $4.20 \mathrm{E}-3$ & 72.0 & 22.0 \\
Treviño et al. (2004) & 9.9 & 15.7 & $0.49 \mathrm{E}-3$ & $4.27 \mathrm{E}-3$ & 106.3 & 21.1 \\
\hline
\end{tabular}

La Tabla 4 presenta características adicionales de los castillos y mampostería requeridos para aplicar el modelo al muro MUR2 elaborado con tabique rojo recocido, el cual fue ensayado ante carga cíclica lateral sin carga vertical (Sánchez, 2009). La Tabla 5 contiene el análisis realizado en 4 incrementos de carga, en el primer incremento ocurre el agrietamiento de los castillos, en el segundo incremento se agrieta la mampostería, en el tercer incremento falla la mampostería y en el cuarto incremento se alcanza la falla de los castillos. Las curvas experimental y numérica son presentadas en la Figura 6a, que muestra la buena aproximación del modelo propuesto.

La Figura 6b muestra los resultados del modelo para el muro M2 elaborado con tabique rojo recocido y ensayado ante carga lateral y esfuerzo vertical (Aguilar, 1997). La Figura 7a contiene la curva media experimental de tres muros elaborados con bloques macizos de jal-cemento sujetos a carga lateral y vertical así como la respuesta numérica (Hernández y Urzua, 2002). En forma similar, la curva media experimental obtenida en el ensaye de carga lateral de 4 muros elaborados con bloques huecos de concreto para un armado específico de los castillos (4 varillas No 3 y estribos No 2) así como la correspondiente curva numérica son presentadas en la figura 7b; la figura 8a muestra los resultados de muros construidos con las mismas piezas cuando se utilizaron armaduras prefabricadas en los castillos (Treviño et al., 2004).

La Figura 8b contiene los resultados obtenidos en el ensaye de un sistema estructural formado por dos muros de tabique rojo recocido sujetos a carga vertical, cuya geometría está definida en la Figura 9 (Ishibashi y Kastumata, 1994). La aplicación del modelo para este sistema no tiene ninguna dificultad adicional y la aproximación es aceptable, aunque no puede garantizarse la correcta sucesión del mecanismo de colapso. Los muros referenciados en la Figura 8b son presentados en la Figura 9. Finalmente, el modelo tiene una buena aproximación para la estructura tridimensional elaborada con muros de tabique rojo recocido probada por Sánchez, Alcocer y Flores (1996) según la Figura 10a.

Tabla 4 Características mecánicas del muro MUR2 (Unidades: ton, m)

\begin{tabular}{cccccc}
\hline \multicolumn{3}{c}{ Castillos $(\boldsymbol{H}=1.75 \mathrm{~m})$} & \multicolumn{2}{c}{ Mampostería } \\
\hline $\boldsymbol{M}_{\boldsymbol{a g r}}$ & $\boldsymbol{M}_{\boldsymbol{y}}$ & $\boldsymbol{V}_{\boldsymbol{a g r}}$ & $\boldsymbol{V}_{\boldsymbol{y}}$ & $\boldsymbol{K}_{\boldsymbol{M} \boldsymbol{1}}$ & $\boldsymbol{K}_{\boldsymbol{M} \boldsymbol{2}}$ \\
\hline 0.31 & 0.84 & 0.35 & 0.96 & 6794 & 1014 \\
\hline
\end{tabular}


Tabla 5 Aplicación del modelo para el muro MUR2 (Unidades: ton, m)

\begin{tabular}{|c|c|c|c|c|c|}
\hline Incremento & Variable & Mampostería & Castillos & Total & Daño \\
\hline \multirow{7}{*}{$1^{\text {ero }}$} & Rigidez $\left(\boldsymbol{K}_{T}\right)=$ & 6794.00 & 511.99 & 7817.97 & \multirow{7}{*}{$\begin{array}{l}\text { Agrietamiento } \\
\text { de los castillos }\end{array}$} \\
\hline & $\Delta_{U N}=$ & & & $1.279 \mathrm{E}-04$ & \\
\hline & $V_{U N}=$ & 0.87 & 0.07 & 1.00 & \\
\hline & $\alpha=$ & 11.43 & 5.41 & & \\
\hline & $\Delta V_{E L}=$ & 4.70 & $\overline{0.35}$ & 5.41 & \\
\hline & $V_{T}=$ & & & 5.41 & \\
\hline & $\Delta=$ & $6.920 \mathrm{E}-04$ & $\Delta_{T}=$ & $6.920 \mathrm{E}-04$ & \\
\hline \multirow{7}{*}{$2^{\mathrm{do}}$} & Rigidez $\left(\boldsymbol{K}_{T}\right)=$ & 6794.00 & 73.28 & 6940.57 & \multirow{7}{*}{$\begin{array}{c}\text { Agrietamiento } \\
\text { de la } \\
\text { mampostería }\end{array}$} \\
\hline & $\Delta_{U N}=$ & & & $1.441 \mathrm{E}-04$ & \\
\hline & $V_{U N}=$ & 0.98 & 0.01 & 1.00 & \\
\hline & $\alpha=$ & $\underline{5.34}$ & 57.37 & & \\
\hline & $\Delta V_{E L}=$ & $\overline{5.23}$ & 0.06 & 5.34 & \\
\hline & $V_{T}=$ & 9.93 & 0.41 & 10.75 & \\
\hline & $\Delta=$ & 7.696E-04 & $\Delta_{T}=$ & $1.462 \mathrm{E}-03$ & \\
\hline \multirow{7}{*}{$3^{\text {ero }}$} & Rigidez $\left(\boldsymbol{K}_{T}\right)=$ & 1014.00 & 73.28 & 1160.57 & \multirow{7}{*}{$\begin{array}{c}\text { Falla de la } \\
\text { mampostería }\end{array}$} \\
\hline & $\Delta_{U N}=$ & & & 8.617E-04 & \\
\hline & $V_{U N}=$ & 0.87 & 0.06 & 1.00 & \\
\hline & $\alpha=$ & $\underline{2.83}$ & 8.70 & & \\
\hline & $\Delta V_{E L}=$ & $\overline{2.47}$ & 0.18 & 2.83 & \\
\hline & $V_{T}=$ & 12.40 & 0.59 & 13.58 & \\
\hline & $\Delta=$ & 7.496E-03 & $\Delta_{T}=$ & 8.957E-03 & \\
\hline \multirow{7}{*}{$4^{\text {to }}$} & Rigidez $\left(\boldsymbol{K}_{T}\right)=$ & 0.00 & 73.28 & 146.57 & \multirow{7}{*}{$\begin{array}{l}\text { Falla de los } \\
\text { castillos }\end{array}$} \\
\hline & $\Delta_{U N}=$ & & & 6.823E-3 & \\
\hline & $V_{U N}=$ & 0.00 & 0.50 & 1.00 & \\
\hline & $\alpha=$ & & 0.74 & & \\
\hline & $\Delta V_{E L}=$ & 0.00 & $\overline{0.37}$ & 0.74 & \\
\hline & $V_{T}=$ & 12.40 & 0.96 & 14.32 & \\
\hline & $\Delta=$ & $5.060 \mathrm{E}-03$ & $\Delta_{T}=$ & $1.402 \mathrm{E}-02$ & \\
\hline
\end{tabular}

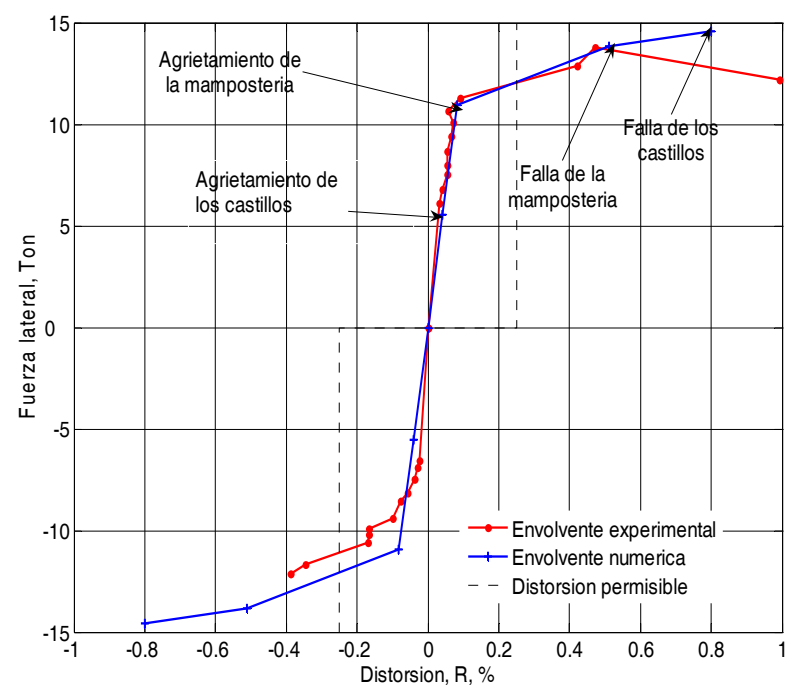

a)

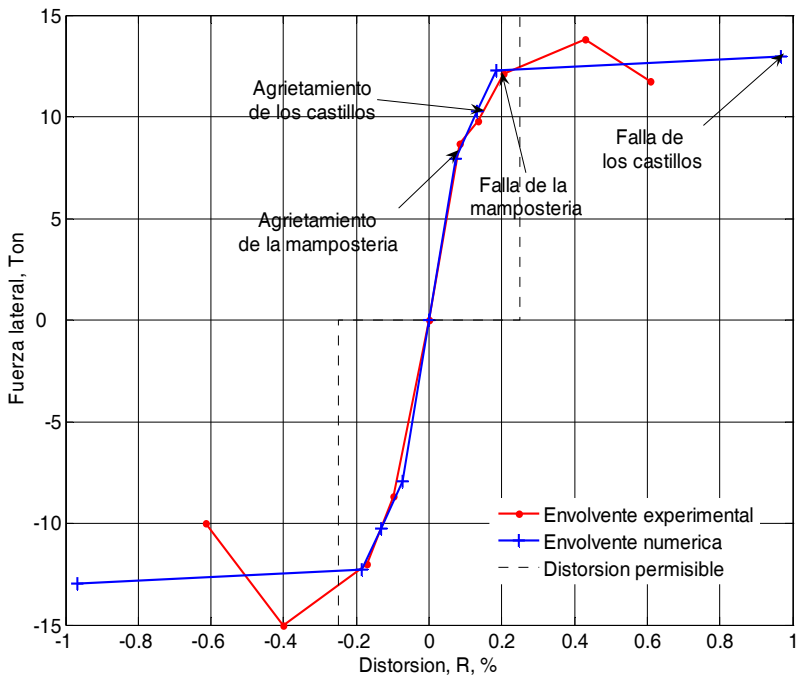

b)

Figura 6: Comparación de resultados para muros elaborados con tabique rojo recocido: a) Muro MUR2 (Sánchez, 2009), b) Muro M2 (Aguilar, 1997) 


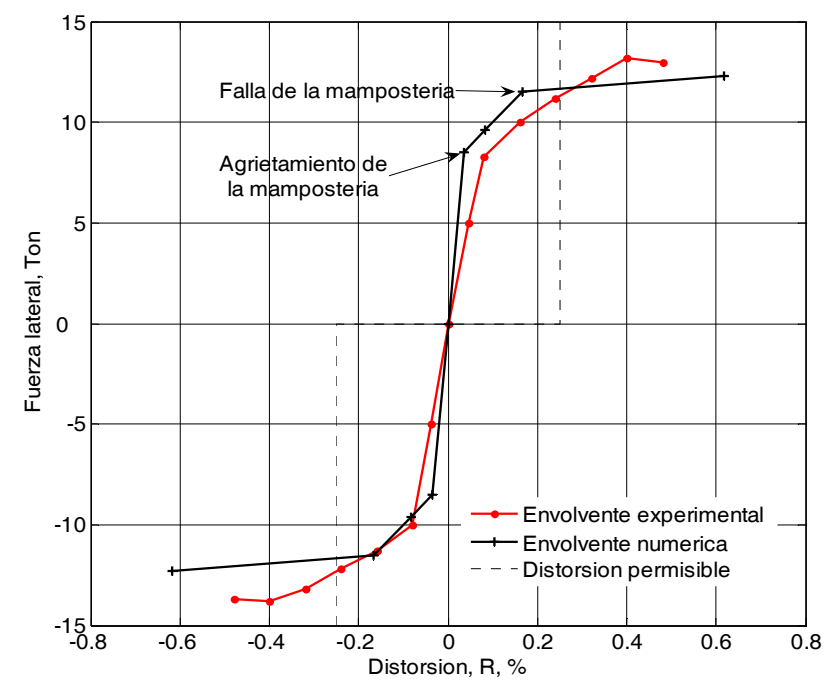

a)

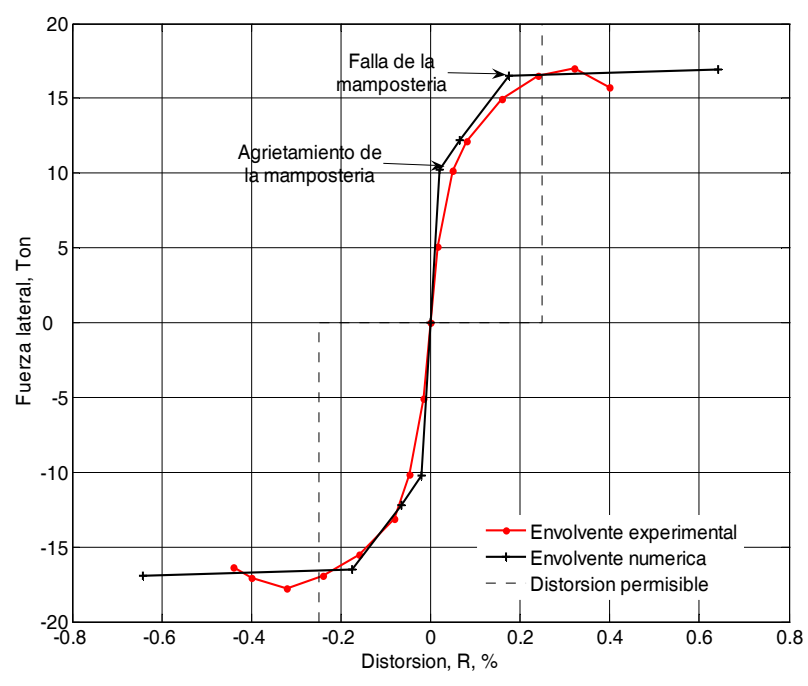

b)

Figura 7: Comparación de resultados para muros elaborados con piezas de concreto: a) Según Hernández y Urzua, 2002; b) Según Treviño et al., 2004 ( $1^{\text {era }}$ serie)

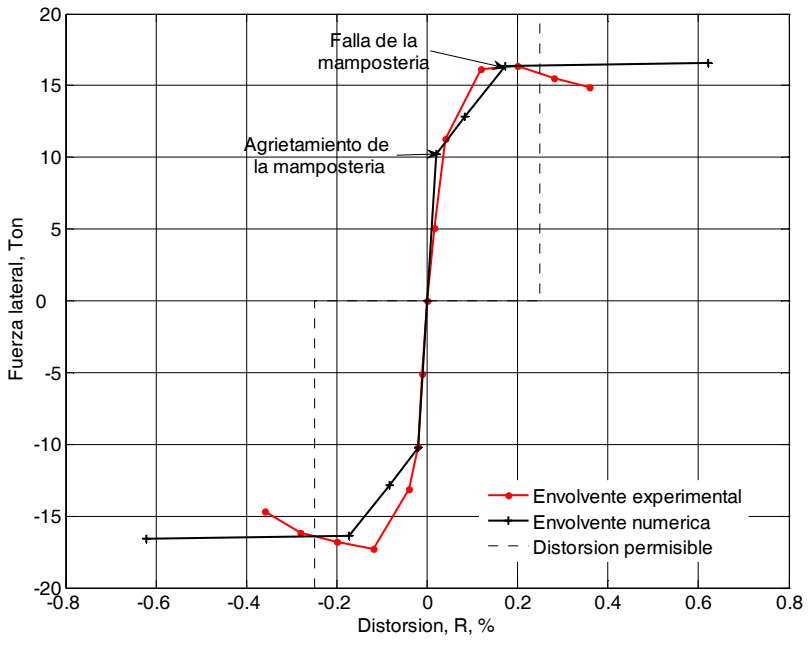

a)

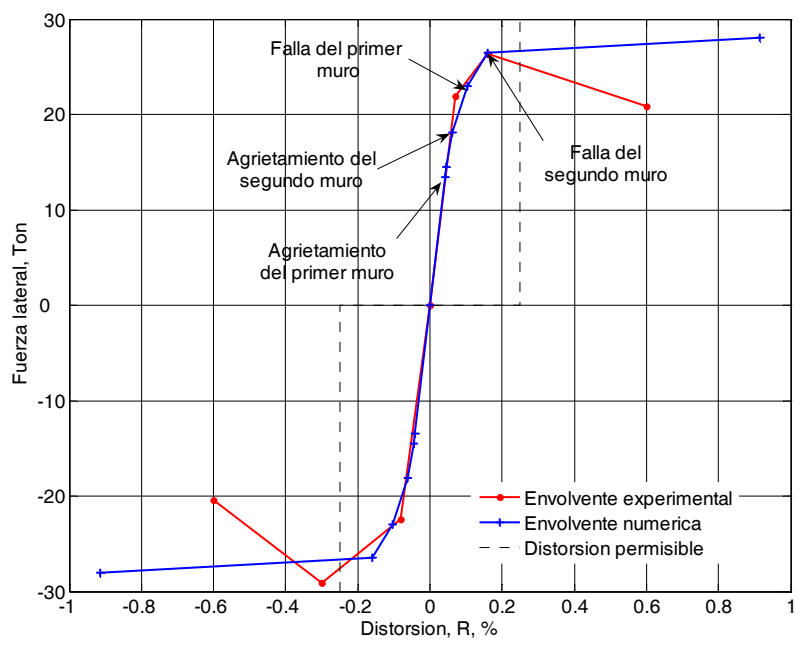

b)

Figura 8: Comparación de resultados: a) Muros elaborados con piezas de concreto ( $2^{\text {da }}$ serie), (Treviño et al., 2004); b) Sistema estructural WWW elaborado con tabique rojo recocido (Ishibashi y Kastumata, 1994)

La aproximación del modelo es evaluada con la Ecuación 21, donde $\boldsymbol{\mu}$ define la razón del valor numérico $\left(\boldsymbol{V}_{\text {num }}\right)$ respecto del valor experimental $\left(\boldsymbol{V}_{\text {exp }}\right)$ de seis parámetros: rigidez elástica, carga y distorsión al agrietamiento, carga asociada a la distorsión permisible (0.25\%), carga asociada a la distorsión de 0.35 \% y carga máxima. En todos los casos presentados, la rigidez elástica fue evaluada entre los dos primeros puntos (positivo y negativo) de las curvas mientras que los parámetros restantes son evaluados en las dos envolventes (positiva y negativa). 
$\mu=V_{\text {num }} / V_{\text {exp }}$

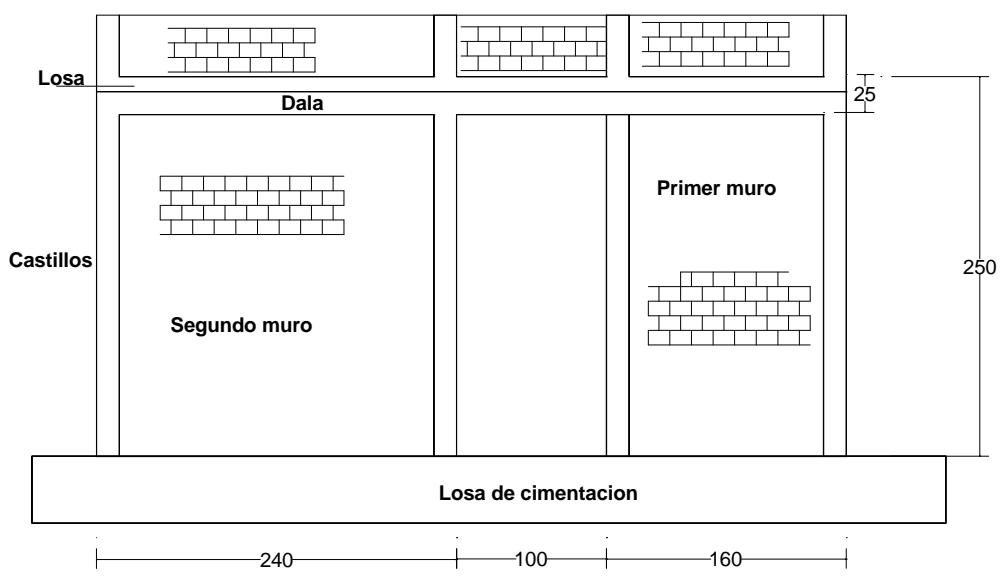

Figura 9: Sistema estructural WWW y definición de primer y segundo muro, dimensiones [cm] (Ishibashi y Kastumata, 1994)

El valor de $\boldsymbol{\mu}$ para las variables de control son presentados en la Tabla 6, la última parte contiene el numero de datos, valor medio, coeficiente de variación y coeficiente de determinación. Los resultados muestran que la rigidez elástica y la distorsión al agrietamiento tienen el mayor coeficiente de variación, $\boldsymbol{C} \boldsymbol{V}=0.35$ con valores medios de 1.31 y 0.66 ; en los parámetros restantes el valor medio está entre 0.97 y 1.00 y el coeficiente de variación no excede $\boldsymbol{C} \boldsymbol{V}=0.08$. El coeficiente de determinación $\boldsymbol{C R}^{2}$, evaluado con la Ecuación 22, tiene valores semejantes a los obtenidos por Riahi, Elwood y Alcocer (2009) excepto para la rigidez elástica.

$$
\begin{aligned}
& C R^{2}=1-\Sigma\left(y_{i}-\hat{y}_{i}\right)^{2} /\left[\Sigma y_{i}^{2}-\left(\Sigma y_{i}\right)^{2} / n\right] \\
& \hat{y_{i}}=b_{o}+b_{1} x_{i}
\end{aligned}
$$

En la Ecuación 22, $\boldsymbol{C R}^{2}$ es el coeficiente de determinación, $\boldsymbol{y}_{\boldsymbol{i}}$ es el valor numérico de la variable en estudio, $\hat{\boldsymbol{y}}_{\boldsymbol{i}}$ es el valor calculado de la variable mediante la Ecuación 23, $\boldsymbol{n}$ es el numero de datos, $\boldsymbol{b}_{\boldsymbol{o}}$ y $\boldsymbol{b}_{\boldsymbol{1}}$ son los parámetros de la ecuación de ajuste lineal de mínimos cuadrados y $\boldsymbol{x}_{\boldsymbol{i}}$ es el valor experimental del parámetro analizado (Canavos, 1999).

De las estructuras aquí referenciadas, dos han sido anteriormente analizadas mediante otro tipo de análisis. Así, los elementos confinantes y la mampostería del sistema estructural WWW fueron modelados por Coral (2004) mediante elementos 1-D; esta misma estructura fue analizada por Álvarez (2000) mediante el método de elemento finito mediante un modelo de grieta giratoria y considerando que la mampostería es homogénea ante esfuerzos de tensión. La estructura 3-D, adicional al análisis de Coral (2004) realizado con los mismos principios del sistema WWW, fue analizada por Zúñiga y Terán (2008) 
mediante el modelo de columna ancha a partir de propiedades mecánicas obtenidas en ensayes previos de muros a escala real.

Tabla 6 Valores de $\boldsymbol{\mu}$ (Resultado numérico/ Resultado experimental)

\begin{tabular}{|c|c|c|c|c|c|c|c|}
\hline Envolvente & $\begin{array}{c}\text { Autor o sistema } \\
\text { estructural }\end{array}$ & $\begin{array}{l}\text { Rigidez } \\
\text { elástica }\end{array}$ & $\begin{array}{l}\text { Carga } \\
\text { agriet }\end{array}$ & $\begin{array}{c}\text { Distorsión } \\
\text { agriet }\end{array}$ & $\begin{array}{c}\text { Carga } \\
\mathrm{R}=0.25 \%\end{array}$ & $\begin{array}{c}\text { Carga } \\
\mathrm{R}=0.35 \%\end{array}$ & $\begin{array}{c}\text { Carga } \\
\text { máxima }\end{array}$ \\
\hline \multirow{8}{*}{ Positiva } & Sánchez (2009) & 0.64 & 0.95 & 0.89 & 1.02 & 1.00 & 1.07 \\
\hline & Aguilar (1997) & 1.13 & 0.91 & 0.88 & 0.99 & 0.94 & 0.94 \\
\hline & Hernández y & & & & & & \\
\hline & Urzua (2002) & 2.07 & 1.02 & 0.43 & 1.03 & 0.94 & 0.95 \\
\hline & $\begin{array}{l}\text { Treviño et al. } \\
(2004) 1_{\sim}^{\text {era }} \text { serie }\end{array}$ & 1.58 & 1.00 & 0.41 & 1.00 & 0.97 & 0.98 \\
\hline & $\begin{array}{l}\text { Treviño et al. } \\
\text { (2004) } 2^{\text {da }} \text { serie }\end{array}$ & 1.41 & 0.90 & 0.50 & 1.00 & 1.10 & 1.03 \\
\hline & WWW & 1.10 & & & 1.05 & 1.12 & 1.06 \\
\hline & 3-D & 1.26 & & & 1.01 & 0.95 & 1.00 \\
\hline \multirow{9}{*}{ Negativa } & Sánchez (2009) & 0.64 & 1.12 & 0.83 & 1.09 & 1.09 & 1.20 \\
\hline & Aguilar (1997) & 1.13 & 0.91 & 0.75 & 0.95 & 0.87 & 0.87 \\
\hline & Hernández y & & & & & & \\
\hline & Urzua (2002) & 2.07 & 0.85 & 0.45 & 0.95 & 0.88 & 0.95 \\
\hline & Treviño et al. & & & & & & \\
\hline & $\begin{array}{l}\text { (2004) } 1^{\text {era }} \text { serie } \\
\text { Treviño et al. }\end{array}$ & 1.58 & 1.00 & 0.43 & 0.97 & 0.95 & 0.94 \\
\hline & (2004) $2^{\text {da }}$ serie & 1.41 & 1.00 & 0.98 & 1.00 & 1.10 & 0.96 \\
\hline & WWW & 1.10 & & & 0.97 & 0.97 & 0.97 \\
\hline & 3-D & 1.26 & & & 1.02 & 1.02 & 1.07 \\
\hline \multirow{4}{*}{$\begin{array}{l}\text { Parámetros } \\
\text { estadísticos }\end{array}$} & Datos & 7 & 10 & 10 & 14 & 14 & 14 \\
\hline & Valor medio & 1.31 & 0.97 & 0.66 & 1.00 & 0.99 & 1.00 \\
\hline & $\mathrm{CV}$ & 0.34 & 0.08 & 0.35 & 0.04 & 0.08 & 0.08 \\
\hline & $\mathrm{CR}^{2}$ & 0.45 & 0.76 & 0.74 & 0.99 & 0.98 & 0.98 \\
\hline
\end{tabular}

Mientras que en el análisis de los modelos WWW y 3-D fue necesario utilizar programas como el DRAIN2D (Coral, 2004), SBETA (Álvarez, 2000) y SAP2000 (Zúñiga y Terán, 2008), que indica cierta complejidad del análisis, la simplicidad del modelo aquí desarrollado permite su implementación en una hoja electrónica de cálculo con resultados similares, ver Figuras 10b y 11.

\section{CONCLUSIONES Y COMENTARIOS}

Bajo la hipótesis que la carga lateral aplicada en muros de mampostería confinada genera exclusivamente una deformación de cortante, un modelo simplificado con un grado de libertad asociado al desplazamiento lateral fue desarrollado, éste supone la falla simultánea de los castillos por efecto cortante cuando alcanzan el momento flexionante de fluencia. Así, la mampostería es sustituida por un elemento 1D ubicado sobre la diagonal del muro con comportamiento (carga lateral-desplazamiento lateral) bi-lineal obtenido en la prueba de tensión diagonal de muretes y considerando el efecto de carga axial. La aproximación de los resultados es aceptable para muros y sistemas estructurales elaborados con las mismas piezas utilizadas en las pruebas de tensión diagonal aquí referenciadas (tabique rojo recocido). Adicionalmente, la aplicación fue extendida a muros elaborados con piezas de concreto para las cuales la 
aproximación disminuye, debido probablemente a la mayor rigidez vertical de la mampostería respecto a la observada en mampostería de tabiques de arcilla.

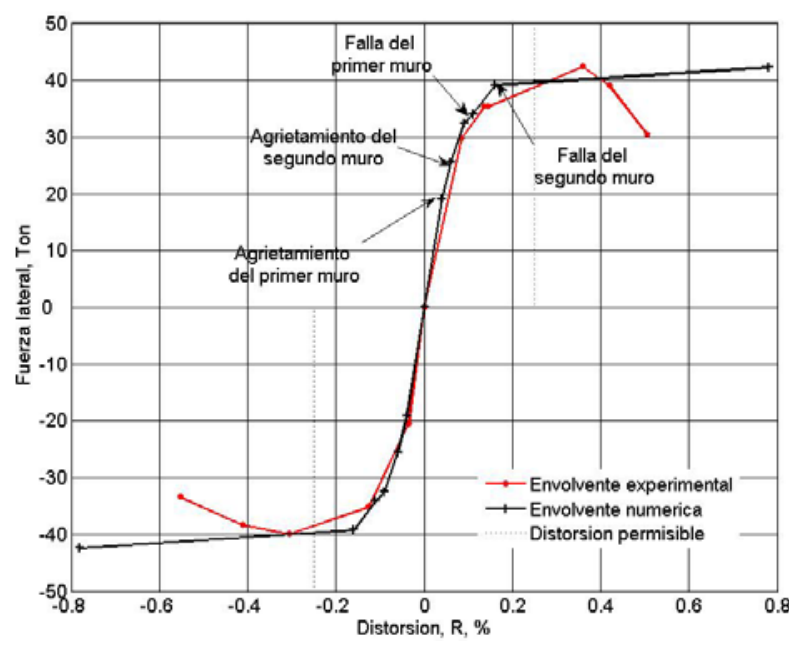

a)

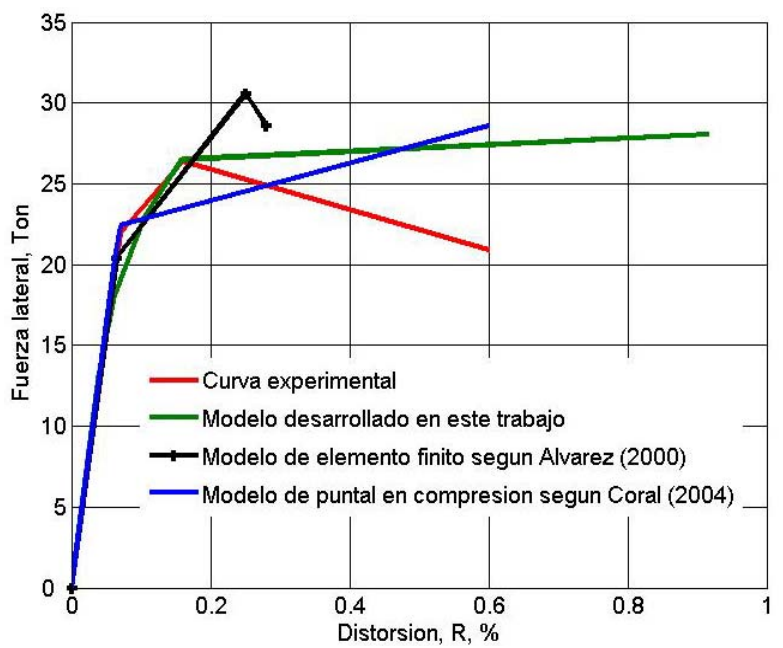

b)

Figura 10: Comparación de resultados: a) De la estructura 3-D (Sánchez, Alcocer y Flores, 1996), b) Del sistema WWW analizado con diferentes modelos (Envolvente positiva)

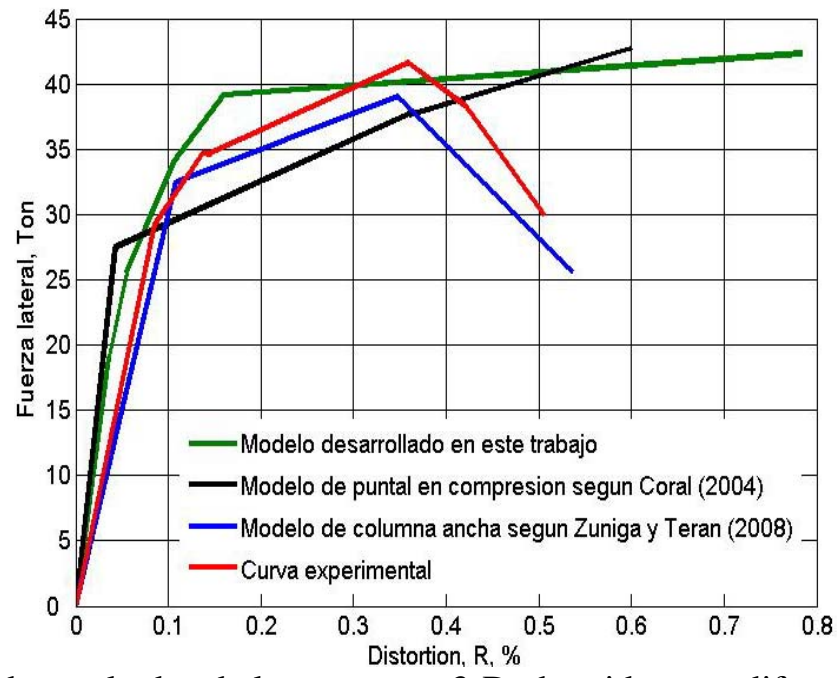

Figura 11: Comparación de resultados de la estructura 3-D obtenidos con diferentes modelos (Envolvente positiva)

Al evaluar los resultados, sólo dos puntos de control, sobre un total de seis, presentan variación importante: la pendiente del tramo inicial y la distorsión al agrietamiento. Sin embargo, la simplicidad del modelo no afecta el nivel de aproximación, similar al obtenido en modelos refinados, y permite evaluar con seguridad la curva de capacidad hasta un rango de distorsión entre $0.35 \%$ a $0.40 \%$, mayor que el valor permisible de $0.25 \%$ definido en NTCM-2004 (2004). Si bien la distorsión puede ser mayor durante un sismo, ésta no puede exceder la máxima distorsión registrada en las pruebas ( $0.6 \%$ en la mayoría de los casos aquí estudiados) para garantizar una resistencia residual del muro. 
El modelo fue desarrollado considerando la situación existente en un entorno geográfico definido, el cual puede presentarse también en estados como Chiapas, Oaxaca, Michoacán y Colima, dicha situación queda definida por los siguientes planteamientos:

1. Se planteó la hipótesis de que las propiedades mecánicas de la mampostería de tabique rojo recocido elaborada en la zona Centro del estado de Guerrero eran diferentes a las referenciadas en las NTCM-2004, lo cual quedó demostrado con los valores del módulo elástico y de cortante obtenidos (Sánchez 2009). Por lo anterior, los autores plantearon que no sería valido utilizar los resultados de los ensayes realizados en la Ciudad de México (con materiales comunes de este lugar) para evaluar el comportamiento de muros construidos en la zona Centro del estado de Guerrero.

2. En la zona mencionada, a diferencia de la Ciudad de México, no existía un Laboratorio de Estructuras para ensayar muros a escala real, por lo cual no había datos para usar o calibrar el modelo de columna ancha propuesto por Zúñiga y Terán (2008).

3. De esta manera, se consideró que los resultados de las pruebas de tensión diagonal en muretes eran la única fuente de información experimental posible a obtener. Esto llevó a la conclusión que dicha información debería ser la base del modelo matemático presentado.

En otro orden, adicional a los tres modelos utilizados (columna ancha según Zúñiga y Terán, 2008; puntales en compresión desarrollado por Coral, 2004; macromodelos con elemento finito presentado por Álvarez, 2000) para comparar los resultados del modelo desarrollado, existen una gran variedad de modelos propuestos para evaluar el comportamiento de la mampostería sujeta a carga lateral, algunos evalúan solamente la envolvente de la respuesta y otros son capaces de obtener el comportamiento histerético. Sin embargo, para una buena elección del modelo utilizado deben considerarse cuatro puntos esenciales: a) facilidad para implementar las leyes de comportamiento en un programa de análisis, b) mínima cantidad de grados de libertad definidos, c) información experimental obtenida en pruebas de especímenes pequeños, d) exactitud de resultados. El elemento resorte de comportamiento bilineal aquí desarrollado cumple ampliamente con los puntos antes mencionados.

Respecto a los límites de aplicación del modelo, la mayoría de información experimental encontrada y utilizada para calibrarlo corresponde a muros de mampostería confinada con relación altura/longitud cercana a uno. Esta misma información fue utilizada para calibrar los distintos modelos referenciados (columna ancha, puntales en compresión, macromodelos con elemento finito), los cuales fueron utilizados posteriormente para analizar estructuras completas donde pueden existir muros esbeltos y otros con castillos intermedios, esta situación puede generar resultados erróneos porque en los primeros hay efectos de flexión y en los segundos, la superficie de falla puede variar respecto a la diagonal. Así, una forma de validar completamente los distintos modelos utilizados es desarrollar pruebas a escala real en muros esbeltos o con castillos intermedios. Sin embargo, es interesante observar que para el sistema estructural WWW y la estructura 3-D, donde existe un muro esbelto con relación altura/longitud igual a 1.56 y otro con relación igual a 1, los resultados son aceptables. Referente al máximo esfuerzo vertical utilizado en las expresiones aquí presentadas, éstas se pueden aplicar en muros con valores de hasta $0.25 \boldsymbol{f}_{\boldsymbol{m}}$ según Sánchez y Mebarki (2009), siendo $\boldsymbol{f}_{\boldsymbol{m}}$ la resistencia a compresión de la mampostería.

\section{AGRADECIMIENTOS}

El desarrollo de esta investigación fue patrocinado por el Programa de Mejoramiento del Profesorado (PROMEP) a través de una beca proporcionada al primer autor. Adicionalmente, se agradece 
el apoyo del Ing. Víctor Flores Vela y la colaboración del personal del CENAPRED, en cuyo laboratorio fueron desarrolladas las pruebas de tensión diagonal aquí referenciadas.

\section{REFERENCIAS}

Aguilar, G (1997), "Efecto del refuerzo horizontal en el comportamiento de muros de mampostería confinada ante cargas laterales”, Tesis de Licenciatura. México: FI-UNAM, 181 p

Álvarez, J (2000), "Estudio analítico sobre el comportamiento no lineal de muros de mampostería confinada con y sin aberturas”, Tesis de maestría. México: FI- UNAM, 147 p

Asociación Colombiana de Ingeniería Sísmica (1994), “Comportamiento sísmico de muros de mampostería confinada” Boletín Técnico 45, Universidad de los Andes, Colombia

ASTM (2007), “Test Method for Diagonal Tension (Shear) in Masonry Assemblages E519-07”, West Conshohocken: Editorial ASTM

Canavos, G (1999), Probabilidad y estadística. Aplicaciones y métodos, primera edición, Editorial McGrawHill, México, pp. 443 - 477. ISBN 968-451-856 0

Castilla, E y A Marinilli (2003), "Experiencias recientes en mampostería confinada de bloques de concreto”, IMME, Vol. 41, No. 2-3, pp. 28-39.

Coral, M (2004), "Revisión de algunas de las hipótesis del método simplificado de análisis sísmico para muros de mampostería confinada”, Tesis de Maestría. México: FI-UNAM, 92 p.

Crisafulli, F, A Carr y R Park, "Analytical modelling of infilled frame structures. A general overview” Bulletin of the New Zealand for Earthquake Engineering, 2000, Vol. 33, no 1

Ewin, B y M Kowalsky (2004), “Compressive behavior of unconfined and confined clay brick masonry”, ASCE Journal of Structural Engineering, Vol. 130, No. 4, pp. 650 - 661

Flores, L (1995), "Estudio analítico de estructuras de mampostería confinada”, Tesis de licenciatura. México: FI-UNAM, 106 p

Gabor, A, E Ferrier, E Jacquelin y P Hameiln (2006), "Analysis an modelling of the in-plane shear behaviour of hollow brick masonry panels" Construction and Building Materials, Vol. 20, No. 5, pp. 308-321

GEG (1989), “Normas Técnicas Complementarias para Diseño por Sismos”, Diario Oficial del Estado de Guerrero, No. 80

Hernández, E y D Urzua (2002), "Pruebas dinámicas de resistencia sísmica de muros de mampostería confinada construidos con materiales pumíticos”, XIII Congreso Nacional de Ingeniería Estructural, Puebla: Editorial SMIE, pp. 337-34

INEGI (2005), II Conteo Nacional de Población 2005

Ishibashi, K y H Kastumata (1994), "A study on nonlinear finite element analysis of confined masonry walls” México: CENAPRED, CI No. 15, 58 p

Kent, D y R Park (1971), “Flexural members with confined concrete”, ASCE Journal, Vol. 97. St. 7

Meli, R y O Hernández (1971), "Propiedades de piezas para mampostería producidas en el Distrito Federal” Informe Técnico, México: II-UNAM, 49 p

Meli, R y G Salgado (1969), “Comportamiento de muros de mampostería sujetos a carga lateral” Informe Técnico 237, México: II-UNAM 
Priestley, M, F Seible y M Calvi (1996), Seismic design and retrofit of bridges. Edit John Wiley \& Sons, New York, 706 p, ISBN 0-471-57998-X,

NTCM-2004(2004), "Normas Técnicas Complementarias para diseño y construcción de estructuras de mampostería”, Gaceta oficial del Distrito Federal

Rodriguez, M (2009), “Confined Masonry Constructions”[Online]. [Cited 6 April 2009]. Available from World Wibe Web: <www. world-housing.net>

Riahi, Z, K Elwood y S Alcocer (2009), "Backbone model for confined masonry walls for performancebased seismic design”, Journal of Structural Engineering, Vol. 135, No. 6, pp. 644 - 654

Sánchez, T, S Alcocer y L Flores (1996), "Estudio experimental sobre una estructura de mampostería confinada tridimensional, construida a escala natural y sujeta a cargas laterales", $X$ Congreso Nacional de Ingeniería Estructural, Editorial SMIE, pp. 909 - 918

Sánchez, S (2009), “Étude expérimental et numérique des murs en maçonnerie confinée chargés dans leur plane. Cas: État de Guerrero (Mexique)”, Thèses de Doctorat. France: Université Paris Est, 196 p.

Sánchez, S y A Mebarki (2009),"Método semi-empírico para estimar la resistencia lateral en muros de mampostería confinada”, Revista de la Sociedad Mexicana de Ingeniería Sísmica, ISSN - 0185092X. Vol. 80, pp. $113-127$

Tomazevic, M (2000), Earthquake-resistant design of masonry buildings, First edition, Editorial Imperial College Press, UK, 268 p. ISBN: 1-86094-066-8.

Treviño, E, S Alcocer, L Flores, R Larrua, J Zarate y L Gallegos (2004), “Investigación experimental del comportamiento de muros de mampostería confinada de bloques de concreto sometidos a cargas laterales cíclicas reversibles reforzados con acero de grados 60 y 42”, XIV Congreso Nacional de Ingeniería Estructural. Sociedad Mexicana de Ingeniería Estructural. Acapulco: Editorial SME

Zabala, F, J Bustos, A Masanet y J Santalucía (2002), “Aspectos del diseño de muros de mampostería encadenada bajo cargas sísmicas”, $X X X$ Jornadas Sul-Americanas de Engenharia Estrutural. Brasil: Editorial Universidad de Brasilia

Zúniga, O y A Terán (2008), "Evaluación basada en desplazamientos de edificaciones de mampostería confinada”, Revista de Ingeniería Sísmica, Vol. 79, pp. 25 -48 


\section{APENDICE A. FACTORES DE REDUCCION DEL ESFUERZO CORTANTE RESISTENTE $\left(v_{m}\right)$}

Un parámetro importante del modelo desarrollado es el esfuerzo cortante resistente $\left(\boldsymbol{v}_{\boldsymbol{r}}\right)$, Ecuaciones seis y nueve, que es una fracción del esfuerzo cortante $\left(\boldsymbol{v}_{\boldsymbol{m}}\right)$ obtenido en la prueba de tensión diagonal, ver Ecuación A1. De esta forma se proponen valores del factor de reducción que son sustituidos en la Ecuación A2 propuesta por Sánchez y Mebarki (2009) para determinar la resistencia ( $\left.\boldsymbol{V}_{\boldsymbol{T}}\right)$ de 34 muros, de los cuales se conocen las características geométrica-mecánicas y la resistencia lateral $\left(\boldsymbol{V}_{\text {exp }}\right)$, las variables del primer término de la Ecuación A2 han sido definidas anteriormente mientras que $\boldsymbol{V}_{\boldsymbol{c}}$ representa la resistencia cortante de los castillos en función del porcentaje de acero, de su sección geométrica y de la resistencia a compresión del concreto.

En la Tabla A1, $\boldsymbol{V}_{\text {num }} / \boldsymbol{V}_{\text {exp }}$ es la razón resistencia teórica contra resistencia experimental. Los valores de $\boldsymbol{F}$ con mejor aproximación son $\boldsymbol{F}=0.676$ para piezas de concreto y $\boldsymbol{F}=0.635$ para piezas de arcilla, con estos valores la razón $\boldsymbol{V}_{\text {num }} / \boldsymbol{V}_{\text {exp }}$ tiene un valor medio $\boldsymbol{\mu}=0.94$ y un coeficiente de variación, $\boldsymbol{C} \boldsymbol{V}=0.17$. Los caracteres entre paréntesis de la primera columna corresponden a la simbología original.

$$
\begin{aligned}
& v_{r}=F v_{m} \\
& V_{T}=A_{d m} \operatorname{sen} \beta\left(v_{r}+f \sigma \operatorname{sen} \beta \cos \beta\right)+2 V_{c r}
\end{aligned}
$$


Tabla A1 Esfuerzo cortante resistente ( $\boldsymbol{v}_{\boldsymbol{r}}$ ) para evaluar la fuerza cortante $\boldsymbol{V}_{\text {num }}$ y razón $\boldsymbol{V}_{\text {num }} / \boldsymbol{V}_{\boldsymbol{e x p}}$

\begin{tabular}{|c|c|c|c|c|c|}
\hline Muro & Autor & Tipo de pieza & $\boldsymbol{v}_{\boldsymbol{m}}, \mathrm{kg} / \mathrm{cm}^{2}$ & $\boldsymbol{v}_{r}, \mathrm{~kg} / \mathrm{cm}^{2}$ & $V_{\text {num }} / V_{\text {exp }}$ \\
\hline $1(1)$ & \multirow{8}{*}{$\begin{array}{c}\text { Castilla y } \\
\text { Marinilli } \\
\text { (2003) }\end{array}$} & Bloque hueco de concreto & 7.64 & 5.16 & 0.91 \\
\hline $2(2)$ & & Bloque hueco de concreto & 7.64 & 5.16 & 0.93 \\
\hline $3(3)$ & & Bloque hueco de concreto & 7.64 & 5.16 & 1.01 \\
\hline $4(4)$ & & Bloque hueco de concreto & 7.64 & 5.16 & 0.85 \\
\hline $5(6)$ & & Bloque hueco de concreto & 7.64 & 5.16 & 0.94 \\
\hline $6(7)$ & & Bloque hueco de concreto & 7.64 & 5.16 & 0.89 \\
\hline $7(8)$ & & Bloque hueco de concreto & 7.64 & 5.16 & 0.90 \\
\hline $8(9)$ & & Bloque hueco de concreto & 7.64 & 5.16 & 0.96 \\
\hline $9(421)$ & \multirow{8}{*}{$\begin{array}{l}\text { Treviño et. al } \\
\text { (2004) }\end{array}$} & Bloque hueco de concreto & 4.31 & 2.91 & 1.09 \\
\hline $10(422)$ & & Bloque hueco de concreto & 4.31 & 2.91 & 1.07 \\
\hline $11(423)$ & & Bloque hueco de concreto & 4.31 & 2.91 & 0.88 \\
\hline $12(424)$ & & Bloque hueco de concreto & 4.31 & 2.91 & 0.96 \\
\hline 13(601) & & Bloque hueco de concreto & 4.31 & 2.91 & 0.98 \\
\hline $14(602)$ & & Bloque hueco de concreto & 4.31 & 2.91 & 0.96 \\
\hline 15(603) & & Bloque hueco de concreto & 4.31 & 2.91 & 0.96 \\
\hline $16(604)$ & & Bloque hueco de concreto & 4.31 & 2.91 & 1.01 \\
\hline 17(MD1) & \multirow{3}{*}{$\begin{array}{l}\text { Hernández y } \\
\text { Urzua (2002) }\end{array}$} & Tabicón jal-cemento & 3.15 & 2.13 & 0.78 \\
\hline 18(MD2) & & Tabicón jal-cemento & 2.85 & 1.93 & 0.77 \\
\hline 19(MD3) & & Tabicón jal-cemento & 5.25 & 3.55 & 0.95 \\
\hline 20(M2) & $\begin{array}{c}\text { Aguilar } \\
\text { (1997) }\end{array}$ & Tabique de barro recocido & 3.90 & 2.48 & 0.82 \\
\hline $21(802)$ & \multirow{3}{*}{$\begin{array}{l}\text { Meli y } \\
\text { Salgado } \\
\text { (1969) }\end{array}$} & Tabique de barro recocido & 5.25 & 3.33 & 0.77 \\
\hline 22(803) & & Tabique de barro recocido & 5.25 & 3.33 & 0.82 \\
\hline $23(804)$ & & Tabique de barro recocido & 12.15 & 7.72 & 0.84 \\
\hline $24(1)$ & \multirow{2}{*}{$\begin{array}{l}\text { Sánchez } \\
\text { (2009) }\end{array}$} & Tabique de barro recocido & 7.10 & 4.51 & 1.32 \\
\hline $25(2)$ & & Tabique de barro recocido & 7.10 & 4.51 & 1.01 \\
\hline $26(3)$ & \multirow{6}{*}{$\begin{array}{l}\text { Asociación } \\
\text { Colombiana } \\
\text { de Ingeniería } \\
\text { Sísmica } \\
\text { (1994) }\end{array}$} & Block hueco de arcilla & 3.50 & 2.22 & 1.23 \\
\hline $27(4)$ & & Tabique de barro recocido & 6.20 & 3.94 & 1.41 \\
\hline $28(5)$ & & Block hueco de arcilla & 11.00 & 6.99 & 0.85 \\
\hline $29(6)$ & & Tabique de barro recocido & 5.00 & 3.18 & 0.71 \\
\hline $30(7)$ & & Tabique de barro recocido & 5.40 & 3.43 & 0.69 \\
\hline $31(8)$ & & Block hueco de arcilla & 8.30 & 5.27 & 1.01 \\
\hline $32(1)$ & \multirow{4}{*}{$\begin{array}{l}\text { Zabala, } \\
\text { Bustos, } \\
\text { Masanet y } \\
\text { Santalucía } \\
\text { (2002) }\end{array}$} & Tabique de barro recocido & 3.16 & 2.01 & 1.17 \\
\hline $33(3)$ & & $\begin{array}{l}\text { Tabique de barro recocido } \\
\text { Tabique de barro recocido }\end{array}$ & 3.16 & 2.01 & 0.78 \\
\hline $34(4)$ & & & 3.16 & 2.01 & 0.69 \\
\hline & & $\boldsymbol{C V}=0.17$ & & \multicolumn{2}{|c|}{$\boldsymbol{\mu}=0.94$} \\
\hline
\end{tabular}

